\title{
Germanica
}

\section{Ailleurs et quête de soi dans Zanzibar d'Alfred Andersch}

Selbstfindung und Versuchung der Ferne im Roman von Alfred Andersch

Sansibar oder der letzte Grund

Distance and self-discovery in Zanzibar by Alfred Andersch

\section{Pascale Avenel-Cohen}

\section{(2) OpenEdition}

\section{Journals}

\section{Édition électronique}

URL : http://journals.openedition.org/germanica/239

DOI : 10.4000/germanica.239

ISSN : 2107-0784

\section{Éditeur}

Université de Lille

\section{Édition imprimée}

Date de publication : 1 juin 2007

Pagination : 13-24

ISBN : 2-913857-19-1

ISSN : 0984-2632

\section{Référence électronique}

Pascale Avenel-Cohen, «Ailleurs et quête de soi dans Zanzibar d'Alfred Andersch », Germanica [En ligne], 40 | 2007, mis en ligne le 16 février 2010, consulté le 06 octobre 2020. URL : http:// journals.openedition.org/germanica/239; DOI : https://doi.org/10.4000/germanica.239

Ce document a été généré automatiquement le 6 octobre 2020.

(c) Tous droits réservés 


\title{
Ailleurs et quête de soi dans Zanzibar d'Alfred Andersch
}

\author{
Selbstfindung und Versuchung der Ferne im Roman von Alfred Andersch \\ Sansibar oder der letzte Grund \\ Distance and self-discovery in Zanzibar by Alfred Andersch
}

Pascale Avenel-Cohen

1 Comme le titre du roman d'Alfred Andersch publié en 1957 l'indique ${ }^{1}$, l'ailleurs est une dimension essentielle dans ce récit. Toutefois, contrairement à ce que l'on pourrait supposer, ce n'est pas sur la côte africaine mais sur celle plus septentrionale et moins exotique de la Baltique, à Rerik, petit port de pêche allemand bien réel où mouillent parfois des bateaux suédois, que se déroule l'essentiel de l'intrigue. C'est le lieu où, durant vingt-quatre heures, se croisent en 1937 les destins de cinq personnages sans lien les uns avec les autres, sinon le parti communiste pour deux d'entre eux, Knudsen, le marin pêcheur seul militant de Rerik encore fidèle à un parti qui l'a semble-t-il abandonné, et Gregor, l'instructeur envoyé par le comité central pour réactiver la cellule locale à l'occasion d'un rendez-vous secret dans l'église Saint-Georges. Mais Gregor a perdu la foi alors qu'il n'avait même pas encore achevé sa formation à l'Académie Lénine de $\mathrm{Moscou}^{2}$ et envisage de profiter de cette dernière mission pour fuir en Suède sur le bateau de Knudsen. Les autres personnages sont plus isolés encore. Judith la jeune juive a quitté la veille sa belle maison de Hambourg où sa mère infirme venait de se suicider pour l'obliger à fuir. Le pasteur Helander, le seul ecclésiastique de Rerik à ne pas avoir cédé au chant des sirènes nazies, sait que ses jours sont comptés. Amputé d'une jambe durant la Première Guerre mondiale, il voit sa plaie se rouvrir sans espoir de guérison du fait d'un fort taux de diabète qui invalide toute opération. Toutefois son énergie se concentre sur le sauvetage d'une statue de novice lisant, œuvre de Barlach ${ }^{3}$ ou d'un de ses élèves, qu'il a lui-même achetée et exposée dans son église malgré l'opposition de sa hiérarchie et que les nazis doivent saisir le lendemain matin. C'est cette statue, symbole de résistance, qui lie les destins de tous les personnages. En effet, Gregor, qui la découvre lors du rendez-vous clandestin dans l'église, ne peut s'empêcher de s'identifier à ce lecteur si concentré et pourtant si 
critique, si bien qu'il décide d'aider Helander et de convaincre Knudsen de la passer en Suède. Pour secourir Judith, rencontrée plus tard dans la taverne du port, il suffira de l'associer à cette opération secrète. La jeune femme et la statue seront sauvées, Gregor renoncera finalement à partir et le pasteur Helander se suicidera en tirant sur les gestapistes venus l'interroger sur la disparition soudaine de la statue, qui l'abattront à leur tour.

2 Si l'on s'en tient aux faits, il est clair que la fuite s'impose comme une problématique évidente du roman: Gregor déserte le parti, Judith fuit la déportation, la statue doit échapper à la destruction, Helander préfère la mort à la torture. C'est un aspect largement retenu par la critique ${ }^{4}$, et pourtant il est loin de restituer la richesse de ce roman et de rendre justice à la quête réelle des personnages, une quête incarnée pour l'essentiel par le jeune mousse anonyme, qui joue un rôle minime - pour ne pas dire insignifiant - dans l'intrigue, mais donne au roman toute sa portée existentielle : celle d'une ouverture vers l'avenir et non simplement d'un refus du présent, la volonté d'un nouveau départ dans un ailleurs, certes lointain mais bien réel, dont Zanzibar est le symbole.

3 En effet, loin d'être polarisé uniquement sur leur point de départ, ce qui est le propre d'une fuite, le mouvement effectué par la majorité des personnages, y compris la jeune juive, est déterminé par leur destination, et c'est ce "gamin $»^{5}$ en retrait de la société qui donne son nom au roman, ce qui en fait un personnage clé, même si son rôle se limite à ramer pour transporter Gregor, Judith et la statue du rivage jusqu'au bateau de Knudsen parce que son patron lui en a donné l'ordre. C'est cet adolescent de quinze ans qui ouvre et ferme le roman. La juxtaposition des deux premières phrases du roman, alors qu'il rêve de partir, et des deux dernières phrases, alors qu'il renonce à rester en Suède, prouve combien le thème du départ et de l'ailleurs sont centraux :

Le Mississippi, ça ce serait quelque chose ! pensait le gamin. Sur le Mississippi il suffirait de chiper un canoë et en route ! à en croire Huckleberry Finn. [...]

Le gamin ne se retourna pas vers la forêt en franchissant l'estacade. Il avança nonchalamment vers le bateau comme si rien ne s'était passé6.

Et de fait, à son niveau, rien ne s'est passé durant ces dernières vingt-quatre heures ; pendant plusieurs heures, lors de son escale sur la côte suédoise, il a caressé l'idée d'échapper à la réalité et de partir vivre en solitaire loin de sa mère si raisonnable, de Rerik et de son interminable apprentissage, et puis est remonté à bord. Pourtant le roman se construit autour de ce gamin et de son aspiration à partir. Dix-neuf des trente-sept chapitres lui sont consacrés, soit un sur deux de manière systématique en commençant par le premier. Chaque chapitre dont le titre est celui des personnages concernés, comme les scènes de théâtre classique, est précédé d'un monologue intérieur intitulé "le gamin", sans rapport direct avec les préparatifs des autres personnages et rédigé en italique pour bien marquer la différence. Même lorsqu'il se mêle aux autres, le gamin ne figure pas dans le titre des chapitres.

En présentant son interprétation personnelle de ce qui se passe autour de lui et d'un monde d'adultes auquel il ne comprend rien tout en insistant sur ses aspirations à quitter Rerik, il joue un rôle de récitant et donne au destin de chaque personnage une cohérence: une quête de l'ailleurs semblable à la sienne, même si les apparences semblent le contredire. Totalement abstrait du monde qui l'entoure et de l'évolution politique de l'Allemagne, par son obsession à imiter son héros Huckleberry Finn ${ }^{7}$ et à 
s'en aller, il donne pourtant au roman non seulement sa trame, mais toute sa signification, en préférant définitivement l'ailleurs à l'utopie et à la fuite.

6 Zanzibar n'est pas à comprendre comme utopique ; tout comme Rerik, c'est un lieu bien réel, un port qui existe, où l'on peut se rendre. C'est pourquoi malgré son admiration sans bornes pour le personnage de Mark Twain et sa volonté de l'imiter, le gamin ne cite pas Huckleberry Finn dans sa liste de trois raisons de quitter son lieu de naissance :

Il fallait quitter Rerik, premièrement parce qu'à Rerik il ne se passait rien, deuxièmement parce que Rerik avait tué son père, troisièmement parce qu'il y avait Zanzibar, Zanzibar au loin, Zanzibar derrière le large, Zanzibar ou la dernière raison.

7 Il l'affirme et le répète, il ne rime à rien de partir pour partir, l'essentiel est d'avoir une destination. La fuite seule ne présente aucune issue ; la mort de son père à laquelle il ne cesse de penser le lui rappelle constamment et c'est bien ainsi qu'il l'interprète. Alors que ce père alcoolique, sans que l'on sache s'il buvait parce qu'il avait peur de naviguer ou parce que l'abîme l'attirait' ${ }^{9}$, s'est noyé lors de l'une de ses sorties en haute mer tandis que tous les autres pêcheurs de Rerik se contentent de cabotage, le gamin refuse cette perspective de fuite dès la première page du roman. Certes, il languit d'ennui dans ce petit port du bout du monde ${ }^{10}$, mais quitter Rerik sans voir d'autre but ne l'entraînerait, il le sait, qu'à disparaître au large, comme son père, sans jamais arriver nulle part. Le départ est fondateur, c'est ainsi que son héros Huckleberry Finn échappe à un quotidien menaçant, mais il ne suffit pas de se soustraire à l'ennui et à la menace, il faut atteindre une destination, construire une autre vie :

On n'est vraiment parti, pensait le garçon, que quand, au-delà de la mer, on a pu toucher terre ${ }^{11}$.

La fuite n'est donc pas l'enjeu de sa quête, même s'il se morfond, même s'il a pris en détestation les autres pêcheurs pour lesquels il n'est que le fils d'un alcoolique qui voulait leur en remontrer en pêchant en haute mer; il ne se contentera pas de monter sur un bateau pour quitter cette côte haïe. Il sait que cela ne le mènerait pas plus loin qu'une simple cachette ou que l'autodestruction qu'avait choisie son père. C'est certainement la raison pour laquelle il renonce à la vie de Robinson qu'il a menée durant quelques heures dans une cabane abandonnée au bord d'un lac en pleine forêt, sur la côte suédoise où accoste Knudsen pour remettre la statue au doyen de Skillinge et débarquer Judith. Cette petite escapade en solitaire ne le conduit au fond pas plus loin que sa cachette habituelle dans la tannerie abandonnée de Rerik, mais elle lui donne la force d'attendre de grandir pour échapper à la tutelle administrative de sa mère et pouvoir partir pour le bout du monde. Il l'avait d'ailleurs bien dit lors de sa treizième intervention :

Il sentait que le grenier n'était plus rien pour lui, ce n'était plus qu'une cachette, et une cachette, c'était trop peu. Ce qu'il fallait, c'était un Mississippi. Se cacher n'avait pas de sens. C'était filer qui avait un sens ${ }^{12}$.

Or, qu'est-ce que le Mississippi sinon la possibilité d'aller toujours plus loin, là où il se passe quelque chose ? Mais dans la forêt de Skillinge, dans une cabane au bord d'un lac, il n'est pas d'avancée possible. Au fond, Skillinge n'est qu'un autre Rerik, il n'offre pas les possibilités illimitées d'un grand fleuve ou d'un port d'échanges comme Zanzibar. Néanmoins, ce premier contact avec l'ailleurs joue un rôle décisif dans l'évolution du personnage, car même si la forêt de Skillinge n'est pas plus ouverte sur le monde que Rerik, elle constitue une étape essentielle dans la quête de soi, comme l'a bien montré Bruno Bettelheim dans son travail sur la Psychanalyse des contes de fée. Symbole bien 
connu, la solitude des bois fournit à cet adolescent la possibilité d'une introspection qui, seule, lui permettra de devenir adulte et de choisir ensuite sa voie avec certitude ${ }^{13}$.

Ce premier contact avec ce qu'il prend d'abord pour un ailleurs permet donc au gamin d'affiner sa définition et de surmonter ses doutes. Il sait désormais que l'ailleurs qu'il recherche n'est pas simplement un point éloigné, c'est un lieu qui doit lui offrir la possibilité d'un destin, foisonner de chances à saisir. Se cacher dans la forêt et imiter son héros américain en devenant trappeur n'est pas une fin en soi. S'il en restait là, il ne ferait que fuir la réalité pour se réfugier dans une vision littéraire qui n'est qu'utopie. Mais le gamin renonce à cette vie illusoire. L'art va certes lui procurer la liberté ${ }^{14}$, mais en tant que guide et non en tant que refuge. Le jeune mousse reconnaît avec bonheur que ses livres avaient raison au moment même où il s'en détache pour se tourner vers la vraie vie, c'est-à-dire lorsqu'il admet qu'il se passe enfin quelque chose et qu'il peut agir, en allant clandestinement chercher un passager ${ }^{15}$.

11 Lorsqu'il revient de son court séjour dans la forêt suédoise, le garçon a franchi un pas décisif, il a renoncé à ses chimères. Cela ne signifie pas qu'il ait renoncé à ses rêves, mais il sait maintenant distinguer l'utopie de la réalité, et il a choisi son camp. L'ailleurs, c'est-à-dire Zanzibar, devient réalité : les livres, les cachettes et les cabanes de forêt ne sont pas des buts ultimes. C'est du reste au moment où il commence à douter de la validité des livres que Zanzibar s'impose à lui comme la troisième raison impérieuse qui le pousse à quitter Rerik, bien avant qu'il n'ait la possibilité d'échapper à son quotidien ${ }^{16}$.

12 Il doit poursuivre dès lors une quête plus radicale, ce qui nécessite qu'il se collette avec la réalité et revienne sur ses pas dans un premier temps, pour échapper à jamais à l'utopie et affronter le monde. C'est la condition nécessaire d'un grand destin. S'il veut pouvoir trouver un ailleurs véritable, il doit tout d'abord renoncer à fuir et apprendre à se mesurer avec Rerik, incarnation du réel. Ce n'est donc pas par hasard qu'Alfred Andersch a situé son roman dans un lieu existant, repérable sur une carte.

13 Entre utopie et ailleurs, entre rêve et réalité, le jeune mousse doit choisir. Or, jusqu'au soir de l'expédition secrète de Knudsen, le gamin ne s'est jamais posé la question avec sérieux. La confrontation avec la possibilité réelle de partir le sort de sa torpeur et le place devant une alternative fondamentale : sera-t-il capable de renoncer à jamais à ses rêves d'enfant pour leur préférer une réalité peut-être décevante? Tel est le nouvel enjeu qui s'offre à lui, le choix entre un destin imaginaire et un destin réel. Quand Knudsen lui apprend qu'il devra lui apporter son aide pour aller chercher un "passager » et lui faire gagner la Suède, l'adolescent ne sait pas encore s'il aura le courage nécessaire pour saisir "sa chance ", ce qui prouve néanmoins qu'il considère dès le départ le réel comme la seule dimension digne de ce nom. C'est à l'aune de sa confrontation avec un ailleurs inconnu et risqué qu'il mesure sa qualité d'homme, comprenant que le rêve n'est pas mieux que la médiocrité routinière à laquelle il entend échapper :

Si nous amenons le passager à bord, se disait-il, alors nous traverserons la mer et cela c'est ma chance.

Du coup il ne pensait plus aux motifs qu'il avait de vouloir partir. Il ne pensait plus à son père, il avait oublié qu'à Rerik il ne se passait rien, et il pensait moins encore à son rêve de Zanzibar. Toutes ses pensées tournaient autour de la chance. Est-ce qu'il saurait en profiter $^{17}$ ? 
Saisir sa chance ne signifie pas seulement partir et trouver un ailleurs, mais aussi être capable d'affronter la vie en adulte. Lorsqu'il s'enfonce dans la forêt, le gamin ne se contente pas de jouer à Huckleberry Finn conformément à ses rêves d'enfant. Il prend le risque de l'ailleurs et jauge ses capacités. Fort de cette expérience, il constate que cet ailleurs-là n'en est pas un, ce n'est tout au plus qu'une utopie comme celle qu'il trouve dans ses livres. Il devra attendre et recommencer. L'ailleurs ne peut pas lui proposer de destin s'il n'est pas apte à l'affronter. De ce point de vue, il n'est pas encore prêt à saisir sa chance et il le sait, mais il est désormais capable de distinguer l'imaginaire du réel. Dans la forêt suédoise, il a pris conscience que son destin était entre ses mains et qu'à l'éloignement de l'ailleurs il devra associer le courage d'agir. En dépit des apparences, ce premier contact avec un ailleurs proche, mais suffisant pour prendre ses distances, est capital.

En partant une première fois, le gamin découvre véritablement qui il est, quels sont ses courages et ses lâchetés, si ses aspirations sont assez fortes pour prendre le risque de tout leur sacrifier. À l'état de rêve ou d'utopie, l'ailleurs ne lui propose que la fuite. Tandis que son père s'enivrait pour prendre le large, il se cache dans une tannerie en ruine pour lire des livres d'aventure qui le dispensent d'agir dans sa vie quotidienne, sorte d'exil intérieur comparable à celui des adultes contraints au silence par le nazisme. Une fois sur la côte suédoise, il ne peut plus se bercer d'illusions. Il doit opérer un choix décisif, non seulement partir ou rester, mais être un rêveur ou un homme d'action. Bien que l'auteur ne donne aucune indication explicite, il est clair que le gamin a opté pour la seconde possibilité ; son retour sur le bateau de Knudsen est un adieu aux utopies bon marché, aux cabanes solitaires, dans l'intention de préparer un vrai grand voyage. Le fait qu'il ne se retourne pas pour regarder derrière lui corrobore cette interprétation. Il préfère monter sur le bateau sous un ciel « sans étoiles », c'est-àdire sans rêve, plutôt que de retourner vivre dans les bois.

Effectivement, comme le signalent les derniers mots du texte, d'un point de vue factuel, "il ne s'est rien passé »: ce petit séjour dans la forêt n'a pas de réalité tangible, c'est une étape, tandis que la mer froide et le ciel gris, c'est-à-dire dénués de fantaisie, sont bien présents. Aussi sombres, glaçants et désespérément matériels soient-ils, c'est eux que choisit l'adolescent, et ce spontanément, sans la moindre tristesse apparente :

Il trouva aisément son chemin; entre les troncs régnait une clarté diffuse, il atteignit de nouveau la route sans vie et après, ce n'était plus loin. Il vit la maison luire à travers les arbres, puis la mer, se glissa derrière les taillis et un rocher jusqu'à l'eau et là il regarda. L'estacade se dressait, ruban gris sur l'eau noire. Le gamin vit que le chalutier était toujours là. Un peu plus loin, la mer était bleue et sombre et froide sous un ciel gris et bas, sans étoiles. Le bateau balançait à peine, il était noir et calme et attendait. Le gamin vit Knudsen sur le pont, assis sur une bouée, et qui fumait.

Le gamin ne se retourna pas vers la forêt en franchissant l'estacade. Il avança nonchalamment vers le bateau comme si rien ne s'était passé ${ }^{18}$.

17 Curieusement, la quête d'un ailleurs d'au moins deux autres personnages, Judith et le pasteur Helander, est liée elle aussi à leurs parents. Si le gamin veut échapper au destin de son père et franchir un pas supplémentaire en accostant quelque part plutôt que de se contenter de flirter avec le large dans une perspective autodestructrice, Judith, elle, comprend sa tentative de fuite par le port de Rerik comme le testament de sa mère. Elle aurait pu en effet accepter la proposition de son banquier qui lui offrait de l'aider par des filières sûres ${ }^{19}$, mais elle refuse catégoriquement. Plus que de fuir, il lui importe 
d'exécuter les dernières volontés de sa mère qui avait de Rerik le souvenir romantique d'un joli petit port lors d'un séjour d'amoureux.

Il est évident que son but est de sauver sa vie en prenant un vapeur pour la Suède, mais elle aurait pu le faire ailleurs avec de meilleures perspectives, car Rerik n'a que peu d'échanges avec l'extérieur. Ce qu'elle est véritablement venue chercher ici n'existe plus et n'a peut-être du reste jamais existé que dans l'imagination poétique de sa mère. Plus encore que ce charmant petit endroit, que ce lieu imaginaire, havre de paix dans l'Allemagne nazie, c'est une réalité fidèle au souvenir de sa mère que Judith espère trouver. Elle escompte débusquer à Rerik une autre Allemagne que celle qui a poussé cette femme à se supprimer, un paysage de paix et d'harmonie. Mais cette quête est d'emblée vouée à l'échec: Rerik n'est pas un lieu d'accueil, pas plus qu'un lieu de départ. Ce n'est ni une utopie, ni un ailleurs. Lorsqu'elle sort de la gare, Judith ne rencontre que la mort, l'exiguïté et le vide sans aucun échappatoire possible ${ }^{20}$, et ce d'autant qu'elle ne connaîtra Rerik que dans l'obscurité toute symbolique du crépuscule et de la nuit. Elle ne pourra pas emporter avec elle l'image d'une Allemagne riante que voulait lui communiquer sa mère comme dernière impression, mais en même temps, elle peut partir sans un regret. Le havre de paix et d'harmonie vers lequel l'envoyait sa mère ne peut se trouver qu'ailleurs.

Le pasteur Helander, lui non plus, n'a pas réussi à trouver à Rerik ce que ses lointains ancêtres scandinaves étaient venus y chercher. Mais il est fort probable que ces " joyeux rêveurs " ${ }^{21}$ venus d'un monde de couleurs se soient contentés, comme la mère de Judith, d'une utopie, tandis qu'il évolue pour sa part dans un monde bien réel, où les ténèbres sont plus obscures que la lumière de son pays d'origine. Aussi Helander rêve-til régulièrement de la Norvège ${ }^{22}$ lorsque sa blessure le fait souffrir, de ce "pays où les maisons étaient en bois et peintes de couleurs vives ${ }^{23}$, sans même se rendre compte qu'il tente ainsi de renouer avec la terre de ses ancêtres. Mais là encore l'ailleurs est utopie. Il est manifeste que la Norvège reste à l'état de rêve, et d'un rêve dont il ne comprend pas l'origine. C'est pourquoi la quête du pasteur Helander se tourne vers un autre espace, un lieu qui soit en même temps un ici et maintenant, c'est-à-dire un monde dans lequel Dieu n'aurait pas disparu en dépit des apparences, un monde dans lequel un signe divin serait malgré tout encore possible. Et c'est ce qui se produit, puisqu'au moment de sa mort Helander voit se dessiner sur le sombre mur d'en face une écriture divine.

La quête de Gregor, le personnage principal puisque le plus proche d'Alfred Andersch en tant que communiste renégat ${ }^{24}$ et le véritable moteur de l'action, n'a certes pas de lien avec ses parents, mais entretient une relation tout aussi étroite avec l'ailleurs. Il ne supporte plus de travailler pour un parti en lequel il a perdu la foi alors que la révolution allemande de 1918 n'était pas encore achevée, et pourtant il reste, porté par le besoin d'avoir une mission à remplir :

Il pensa : à supposer qu'il y ait encore des missions, celles du Parti sont les seules auxquelles il vaille encore la peine de croire. Et s'il survenait un monde où les missions n'existent pas? Un doute épouvantable l'envahit: pouvait-on vivre sans une mission ${ }^{25}$ ?

21 Et pourtant, Gregor envisage sérieusement de quitter l'Allemagne et de profiter de cette dernière mission du comité central qui l'a mené à Rerik pour déserter et passer en Suède sur le bateau de Knudsen. Pour partir, pour aller ailleurs, il semble prêt à renoncer à ce qui constitue sa raison de vivre depuis au moins vingt ans : avoir une 
mission à remplir. Et pourtant, au final Gregor ne part pas, non pas qu'il change d'avis, mais parce qu'il s'aperçoit que ce qu'il recherche n'est pas un éloignement géographique ou un dépaysement, mais une raison de vivre que la traversée de la Baltique ne saurait lui apporter. Confronté à la statue du novice au livre, Gregor découvre la possibilité d'une foi sincère qui puisse être critique ${ }^{26}$. Il trouve ainsi légitimés ses propres doutes sans invalider sa soif de mission à remplir. Foi et liberté ne seraient pas nécessairement antinomiques. Fort de cette découverte qui le bouleverse, il envisage pour la première fois la possibilité d'une action privée qui se substitue aux missions du Parti. Soudain libéré de toute contrainte, il ne ressent plus le besoin de placer la mer entre le KPD et lui. Il découvre que l'ailleurs auquel il aspirait n'était rien d'autre qu'un espace de liberté :

Et moi, qu'est-ce que je veux? Je veux sortir de mon coin et filer quelque part où l'on puisse encore réfléchir, chercher si cela a encore un sens de croire au Parti ${ }^{27}$.

À partir de là, il comprend qu'il est inutile de fuir et que c'est en lui qu'il doit puiser des raisons d'agir :

Le jour vide s'était empli [...] Il réalisa soudain qu'il avait oublié le Parti et qu'il était libre, libéré par des choses qu'il est impossible d'expliquer : des tours et du calme, $\mathrm{du}$ jais et une trahison. Tout cela était plus fort que le Parti : c'était cela et non le parti qui avait retenu ses pas tournés vers la fuite, si bien qu'il errait maintenant sans but, par jeu presque, dans le port de Rerik ${ }^{28}$.

Ce renoncement à l'ailleurs est synonyme d'une prise de confiance en soi, d'une personnalité qui se redécouvre et apprend la liberté. En refusant de partir, Gregor s'assume et cesse de se considérer comme un traître. Dès lors, il recouvre son intégrité et ne ressent plus le besoin de fuir. L'ailleurs auquel il aspirait était en lui.

Dans ce court roman, l'ailleurs est indissociable de la quête de soi et de la liberté. Que ce soit pour y renoncer, comme Gregor, pour partir faute d'autre solution, comme Judith, ou pour prendre l'élan nécessaire à un grand départ ultérieur comme le jeune mousse, le contact avec l'ailleurs fortifie tous les personnages. C'est en caressant l'idée de partir qu'ils trouvent tous le courage d'affronter la réalité.

L'ailleurs n'est pas une utopie dans laquelle se réfugier. C'est pour renoncer à cette dimension que Gregor et le gamin décident de rester, ne serait-ce qu'un temps. L'ailleurs véritable doit être une destination d'adulte responsable et sûr de soi, capable d'affronter le réel, de se mesurer au réel et de prendre son destin en main grâce à une lucidité qu'il ne peut acquérir que par un premier contact avec l'extérieur.

\section{NOTES}

1. Alfred Andersch: Sansibar oder der letzte Grund, Zurich, Diogenes, 1957. Dans la suite, les références à cette édition seront abrégées en $\mathrm{S}$, suivi du numéro de page.

2. «Vielleicht hatte der Verrat schon früher begonnen, vielleicht schon in einem plötzlichen Ermüden während einer Vorlesung in der Lenin-Akademie, auf die der Jugendverband Gregor geschickt hatte, für seine organisatorischen Verdienste in Berlin » (S 23).

3. L'œuvre d'Ernst Barlach fut condamnée par les nazis comme « art dégénéré ». 
4. Cf. Walter Heist : «Flucht in die Arktis : über Alfred Andersch », Merkur, Deutsche Zeitschrift für europäisches Denken, Münich, 1970, p. 446-458 ou Hans Geulen: «Probleme der dargestellten Erfahrung des 'deutschen Irrtums' », in: Hans Wagener: Gegenwartsliteratur und Drittes Reich. Deutsche Autoren in der Auseinandersetzung mit der Vergangenheit, Stuttgart, Reclam, 1977, p. 205-221.

5. Ce personnage ne porte pas d'autre nom que « le gamin » (der Junge).

6. Alfred Andersch : Zanzibar, traduit de l'allemand par Jean-Robert Hennion, Paris, Seuil, 1960, p. 7 et 220 (S 7 et 147). Il sera constamment fait référence à cette traduction, abrégée en $\mathrm{Z}$, suivi du numéro de page.

7. Mark Twain, Les aventures de Huckleberry Finn, 1885.

8. Z 116 ; « Man mußte Rerik verlassen, erstens, weil in Rerik nichts los war, zweitens, weil Rerik seinen Vater getötet hatte, und drittens, weil es Sansibar gab, Sansibar in der Ferne, Sansibar hinter der offenen See, Sansibar oder den letzten Grund. » (S 77).

9. Hésitant entre les deux possibilités, le gamin ne parvient pas à trouver de réponse à ses interrogations (S 16).

10. L'expression n'est pas métaphorique, c'est bien ici l'ennui qui a conduit son père à mourir pour tenter d'échapper à cette épouvantable routine (S 35), et l'ennui est la raison première qu'évoque le gamin pour partir: «Es war tatsächlich überhaupt nichts los. Niemals wird hier irgend etwas mit mir geschehen, dachte der Junge, während er den herbstgelben, lanzettförmigen Weidenblätter nachsah, wie sie auf der Treene langsam abschwammen » (S 9).

11. $Z 8$; «Erst dann ist man weg, dachte der Junge, wenn man hinter der offenen See Land erreicht $\gg(\mathrm{S} 7)$.

12. Z 116 ; «Er spürte, daß der Speicher nichts mehr für ihn war, er war nur ein Versteck, und ein Versteck war zu wenig, was man brauchte, das war ein Mississippi. Sich Verstecken hatte keinen Sinn, nur Abhauen hatte einen Sinn » (S 77).

13. Cf. Bruno Bettelheim : Kinder brauchen Märchen, traduit de l'américain par Liselotte Mickel et Brigitte Weitbrecht, Munich, dtv, 1977, à propos du conte Les deux frères, p. 109 : «Der Wald, in den sie zusammen gehen, um sich darüber einig zu werden, daß sie ein eigenes Leben führen wollen, steht symbolisch für den Ort, an dem man sich dem inneren Dunkel stellt und es durchdringt, an dem die Unsicherheit im Blick darauf, wer man ist, vergeht und an dem man zu verstehen beginnt, wer man sein möchte».

14. Cf. Volker-B. Hetzel : «Die Kunst als Möglichkeit der Freiheit im Werk Alfred Anderschs", Revue des langues vivantes, $1969, \mathrm{n}^{\circ} 35$, p. 531-549 et 580-96, ici p. 583-584.

15. «Er hob das Brett hoch, unter dem er seine Bücher versteckt hatte, da lagen sie, und zum erstenmal betrachtete er sie mit einem Gefühl des Mißtrauens. Er hatte den Tom Sawyer und die Schatzinsel und den Moby Dick und Kapitän Scotts letzte Fahrt und Oliver Twist und ein paar Karl-May-Bände, und er dachte : die Bücher sind prima, aber sie stimmen alle nicht mehr, so, wie es in den Büchern zugeht, so geht es heute nicht mehr zu » (S 76) et «Und plötzlich dachte der Junge : dann stimmen die Bücher ja doch noch, dann gibt es ja auch heute noch solche Sachen, wie sie im Huckleberry Finn und in der Schatzinsel und im Moby Dick erzählt werden. Toll, dachte der Junge, und so was macht Knudsen » (S 94).

16. Cf. Volker-B. Hetzel : « Die Kunst als Möglichkeit der Freiheit im Werk Alfred Anderschs », op. cit., p. 584.

17. Z 168 ; «Wenn wir den Passagier an Bord kriegen, dachte er, dann fahren wir über die See, und das ist die Chance für mich. Nie hätt'ich gedacht, daß ich mal 'ne Chance kriegen würde. Jetzt, da er die Chance spürte, dachte er übrigens nicht mehr an die Gründe, warum er weg wollte. Er dachte nicht mehr an seinen Vater, er hatte vergessen, daß in Rerik nichts los war, und am allerwenigsten fiel ihm sein Traum von Sansibar ein. Alle seine Gedanken kreisten um die Chance, und ob es ihm gelingen würde, sie auszunutzen » (S 112). 
18. Z 220 ; «Er fand den Weg leicht, zwischen den Stämmen herrschte ein diffuses graues Licht, wieder kam er über die leblose Straße, und dann war er nicht mehr weit. Er sah das Haus durch den Wald schimmern und dann die See, und er machte sich hinter Unterholz und einem Felsen bis ans Meer heran und spähte hinaus. Der Steg lag als graues Band über dem schwarzen Wasser. Der Junge sah, daß der Kutter noch immer dalag. Etwas weiter weg war das Meer blau, dunkelblau und kalt lag es unter einem grauen, einförmigen Himmel ohne Sterne. Der Kutter bewegte sich kaum, er war schwarz und still und wartete. Der Junge konnte sehen, daß Knudsen auf Deck saß, er saß auf der Wassertonne und rauchte. Der Junge blickte nicht mehr in den Wald zurück, als er den Steg betrat. Er schlenderte auf das Boot zu, als sei nichts geschehen » (S 146).

19. «Heise hatte verschiedene ausgezeichnete Fluchtwege vorgeschlagen, aber Judith hatte dazu nur eigensinnig den Kopf geschüttelt. Mama war gestorben, damit sie, Judith, nach Rerik gehen könne. Es war ein Testament und sie hatte es zu vollstrecken » (S 18).

20. «Sie hatte sich Rerik ganz anders vorgestellt. Klein und bewegt und freundlich. Aber es war klein und leer, leer und tot unter seinen riesigen roten Türmen. [...] Auf jeden Fall waren Türme, die sich um Mamas Gifttod nicht kümmerten, das fühlte Judith. [...] Und es lag kein Dampfer, kein noch so kleiner Dampfer im Hafen » (ibid.).

21. «fröhliche Träumer» (S 10).

22. S 138 .

23. $\mathrm{Z} 13 ; \mathrm{S} 10$.

24. Cf. Margarete Littler: "Der einstweilige Genosse : Alfred Andersch and the KPD ", New German Studies (NGS), 1988-1989, vol. 3, p. 225-235.

25. Z 57 ; «Er dachte : wenn es überhaupt noch Aufträge gibt, dann sind die Aufträge der Partei die einzigen, an die zu glauben sich noch lohnt. Wie aber, wenn es eine Welt ganz ohne Aufträge geben sollte? Eine ungeheuere Ahnung stieg in ihm auf: konnte man ohne einen Auftrag leben?» (S 39).

26. «Kann man das : ein junger Mönch sein und sich nicht von den Texten überwältigen lassen? Die Kutte nehmen und trotzdem frei bleiben? Nach den Regeln leben, ohne den Geist zu binden? Gregor richtete sich auf. Er war verwirrt. Er beobachtete den jungen Mann, der weiterlas, als sei nichts geschehen. Es war aber etwas geschehen, dachte Gregor. Ich habe einen gesehen, der ohne Auftrag lebt. Einen, der lesen kann und dennoch aufstehen und fortgehen. Er blickte mit einer Art Neid auf die Figur» (S 40).

27. Z 68 ; «Und ich, was will ich? Aus meinem Winkel raus und irgendwohin, wo man nachdenken kann, darüber nachdenken, ob es noch einen Sinn hat, an die Partei zu glauben » (S 45).

28. Z 92 ; « Der leere Tag hatte sich gefüllt [...] Er begriff auf einmal, daß er die Partei vergessen hatte und daß er frei war, befreit durch Dinge, die sich überhaupt nicht fassen ließen : Türme und Gelassenheit, Windschwarz und Verrat. Sie waren stärker als die Partei : sie waren es, nicht die Partei, die seinen schon zur Flucht gewendeten Fuß innehalten, so daß er sich jetzt ziellos, fast spielerisch am Hafen von Rerik herumtrieb» (S 60-61).

\section{RÉSUMÉS}

Comme le titre du roman l'indique, alors que l'action se déroule uniquement dans un petit port allemand de la mer Baltique, l'ailleurs est une dimension essentielle de ce récit. Même le personnage de la jeune juive obligée de quitter l'Allemagne pour échapper à la barbarie nazie ne 
se contente pas de fuir géographiquement: en venant à Rerik, Judith cherche à retrouver l'atmosphère romantique des jours heureux où ses parents avaient effectué un voyage en amoureux sur ces rives. De la même façon, Gregor, le cadre du parti communiste venu réorganiser la cellule locale, mais en pleine crise de conscience, préférera renoncer à monter sur le bateau qui pourrait le mettre à l'abri et lui donner accès à une vie nouvelle sans lien avec son passé. Rester lui permet au contraire une remise en question fondamentale de ses convictions. C'est ainsi qu'il pourra trouver véritablement sa voie plutôt que de se contenter d'un simple éloignement. La problématique soulevée par le jeune garçon fasciné par les romans d'aventure est assez voisine. Mais s'il refuse de partir, c'est parce que son premier contact avec l'ailleurs lui apprend à distinguer l'utopie du réel pour lequel il se décide finalement. Tous réunis dans ce port, ouverture sur un ailleurs géographique réel, la plupart des personnages refuseront de partir. Ils font ainsi le choix de la confrontation avec la réalité plutôt que celui d'un ailleurs qui ne saurait finalement les satisfaire mais leur permet d'achever leur quête de soi.

Wie der Titel des Romans zeigt, ist die Ferne eine wesentliche Komponente des Textes, obwohl die Geschichte sich ausschließlich in einem kleinen Hafen an der Ostsee abspielt. Sogar Judith, die junge Jüdin, die Deutschland verlassen muss, um der Nazi-Barbarei zu entgehen, beschränkt sich nicht darauf zu fliehen : In Rerik versucht sie die romantische Stimmung einer glücklichen Reise ihrer Eltern wieder zu finden. Gregor, der Instrukteur des Zentralkomitees, der die Partei in Rerik neu strukturieren soll, aber von Zweifeln überwältigt wird, verzichtet darauf, im Boot von Knudsen zu fliehen, um ein neues sicheres Leben in Schweden zu beginnen. Indem er bleibt, unterzieht er seine Überzeugungen einer radikalen Prüfung. So findet er seinen Weg, statt sich nur geographisch zu entfernen. Die Problematik des Jungen, der von Abenteuerromanen fasziniert ist, ist ähnlich. Auch er weigert sich zu fliehen, weil sein erster Kontakt mit der Ferne ihn lehrt, wie man Utopie von Realität unterscheidet, so dass er sich für letztere entscheidet. Fast alle Figuren, die sich in diesem Hafen treffen, der die Perspektive der Ferne in der realen Welt symbolisiert, verzichten schließlich darauf, wegzugehen. Sie entscheiden sich auf diese Weise für die Konfrontation mit der Realität anstatt für eine Ferne, die sie nicht zufrieden stellen kann, aber die allein ihre Selbstfindung ermöglicht.

As the novel's title indicates, while the plot is only taking place in a small German port on the Baltic Sea, the distance is an essential dimension of this narrative. Even the character of the young Jewish girl forced to leave Germany to escape from the Nazi barbarity does not only leave geographically: by coming to Rerik, Judith tries to find back the romantic atmosphere of the happy days when her parents had done a romantic journey on these shores. In a similar way, Gregor, the Communist party's leader who came to reorganize the local office but meanwhile in the middle of a crisis will prefer renounce to embark on the boat that could take him to a safe place and bring him a new life that would not be connected with the past. Thus he will be able to really find his path rather than only accepting a simple distance. The problematic rose up by this young man who is fascinated by adventure novels is quite close. But if he refuses to leave, its is due to his first contact with the distance that teaches him to distinguish utopia from the real for which he eventually decides on. All gathered in this port, opening to a real geographical distance, most of the characters will refuse to leave. They thus choose to face with reality rather than the one of a distance that could not eventually content them but enable them to achieve their selfdiscovery.

\section{INDEX}

Mots-clés : départ, voyage

oeuvrecitee Sansibar oder der letzte Grund, Zanzibar 
AUTEURS

PASCALE AVENEL-COHEN

Université Charles-de-Gaulle - Lille 3 